\title{
A Digital Method for the Discrimination of Neutrons and $\gamma$ Rays With Organic Scintillation Detectors Using Frequency Gradient Analysis
}

\author{
Guofu Liu, Malcolm J. Joyce, Xiandong Ma, and Michael D. Aspinall
}

\begin{abstract}
A digital method for the discrimination of neutron and $\gamma$-ray events from an organic scintillator has been investigated by using frequency gradient analysis (FGA) based on the Fourier transform. Since the scintillation process and the photomultiplier tube (PMT) anode signal are often very noisy, most pulse-shape discrimination methods in a scintillation detection system (e.g., the charge comparison (CC) method or pulse gradient analysis (PGA)) using time-domain features of the signal depend greatly on the associated de-noising algorithm. In this research, the performance of the new FGA method and the PGA method have been studied and compared on a theoretical basis and then verified by time-offlight (TOF). The frequency-domain features extracted by the FGA method exhibit a strong insensitivity to the variation in pulse response of the photomultiplier tube (PMT) and can be used to discriminate neutron and $\gamma$-ray events in a mixed radiation field. It is shown that the FGA method results in an increased figure of merit (FOM) which corresponds to a reduction in the area of overlap between neutron and $\gamma$-ray events. The FGA method has the potential to be implemented in current embedded electronic systems to provide real-time discrimination in standalone instruments.
\end{abstract}

Index Terms $-\gamma$ rays, digital discrimination, frequency gradient analysis, neutron, organic scintillators, pulse gradient analysis, time of flight.

\section{INTRODUCTION}

$\mathbf{O}$ RGANIC scintillation detectors have often been used for the detection and spectroscopy of a wide assortment of radiations. For the vast majority of liquid organic scintillators, the composite yield curve consists of two exponential decays - the fast and slow components of the scintillator. Because the fraction of light that appears in the slow component often depends on the nature of the exciting particle, one can use this dependence to differentiate different kinds of radiation. Certain organic scintillators, including stilbene and a number of commercial liquid scintillators, are particularly good exponents of this phenomenon that is exploited via pulse shape discrimination (PSD). This is because of the large differences in the slow component induced by different radiations in these media. When organic scintillators are used as neutron detectors, PSD is an essential requirement because all neutron fields coexist with

Manuscript received July 17, 2009; revised November 12, 2009; accepted January 26, 2010. Date of current version June 16, 2010. This work was supported in part by the Engineering and Physical Sciences Research Council (EPSRC) and the Royal Academy of Engineering.

The authors are with the Department of Engineering, Lancaster University, Lancaster LA1 4YR, U.K. (e-mail: g.liu1@lancaster.ac.uk; m.joyce@lancaster.ac.uk; xiandong.ma@lancaster.ac.uk; m.aspinall@lancaster.ac.uk).

Digital Object Identifier 10.1109/TNS.2010.2044246 an associated $\gamma$-ray component, arising as a result of scattering reactions of the neutrons with materials in the environment and as direct by-products of the primary reaction producing the neutron field [1].

A number of techniques for PSD have been investigated with varying degrees of success. The most popular of these are the charge comparison method [2] and the zero crossing method [3]. Both of these methods were originally implemented in analogue electronics, often in dedicated instrumentation modules or nuclear instrument modules (NIMs). More recently, both of these methods have since been implemented in the digital domain as digital electronic platforms have become available with the requisite speed and cost to make this possible. These methods have become the industrial standards to be used to compare with other new proposed discrimination methods, such as the correlation method [4] and the curve-fitting method [5].

In recent years, artificial neural networks (ANN) and fuzzy systems (FS) have emerged as mature technologies, with successful applications across many fields. They are particularly effective as pattern recognition tools, and on this basis ANN and FS can be used to classify neutron and $\gamma$-ray events from the measurements performed by organic liquid scintillation detectors. In particular, G. Liu et al. [6] used a classic back-propagation (BP) neural network to process the shape of light pulses in an organic liquid scintillator; while D'Antone [7] proposed an approach based on fuzzy logic for pulse-shape discrimination in a single-layer scintillator detector.

In order to take advantage of capabilities offered by fast digital electronics, B.D'Mellow et al. [8] presented a discrimination approach that is computationally simple, referred to as pulse gradient analysis (PGA). This technique provides real-time, digital characterization of environments where neutrons and $\gamma$ rays co-exist. The performance of the PGA method has been compared against a digital implementation of the conventional charge comparison method which demonstrated the PGA method provides an improvement in discrimination ability. Very recently, a dedicated portable instrument has been developed that provides real-time discrimination of mixed fields that is based on the PGA algorithm deployed on Xilinx Virtex $5^{\circledR}$ field-programmable gate array [9].

All of the above pulse-shape discrimination methods use time-domain features of the signal, e.g., the implementation of charge comparison method generally relies upon the integration of the pulse over two different intervals and that of PGA method is based on the comparison of the relative heights of samples in the trailing edge of the pulse. Since the scintillation process 
and the photomultiplier tube (PMT) anode signal are often very noisy and time-domain features are naturally highly dependent on the signal amplitude at specific times, these pulse-shape discrimination methods can have a great dependency on the de-noising algorithm. For example, the results of simulation of PGA show that the efficacy of this method in the presence of significant PMT variation in pulse response is reduced significantly [8]. In the absence of this source of variance to the signals, the FOM of PGA is theoretically infinite.

Based on the above considerations, some researchers have begun to consider PSD methods operating in the frequency domain. Compared with time domain, the methods developed in frequency domain are more robust to the natural variance in the pulse response arising from the PMT. There are two popular mathematical approaches to such spectral analysis: the Fourier transform and the wavelet transform. The Fourier transform is usually considered to be the best transformation between time and frequency domains because it is time-shift invariant; while the wavelet transform is an efficient tool with which to analyze non-stationary signals on a time-frequency scale.

S. Yousefi et al. [10] has recently proposed a new PSD method based on the wavelet transform which is able to detect neutrons and $\gamma$ rays in liquid scintillators. In this method, because the features are extracted at scales 512 and 1024 which are related to two low-frequency components, the discrimination algorithm is less sensitive to high-frequency components that the result of the PMT-borne variation in pulse response which is present in the frequency spectrum of pulses. Experimental results show that compared to PGA algorithm, the wavelet-based method provides an improvement in reducing the overlap between neutron and $\gamma$-ray events reflected by an increase in the FOM. However, the overhead of calculation of the wavelet-based PSD method is heavier than that of PGA algorithm and thus it is not as suitable for real-time discrimination. Furthermore, its efficacy is likely to decline at processing speeds consistent with current embedded systems, as opposed to the relatively unlimited processing head room of the digital capture oscilloscopes on which it has been developed.

In this paper, a new PSD method is proposed based on the Fourier transform, termed as frequency gradient analysis (FGA). This exploits the difference between the zero-frequency component and the first frequency component of Fourier transform of the acquired signal. The objective of this method is to combine the advantage of insensitivity to pulse variation associated with spectral analysis with that of real-time implementation of the PGA algorithm. A comparison of the performance of the FGA method with the PGA method has been made on a theoretical basis and verified by time of flight (TOF). It is shown that FGA method not only increases the FOM, as a result of reducing the overlap area between neutron and $\gamma$-ray events, but that it can also be implemented in real-time as is the case for the PGA method. The relevance of these issues in the current climate associated with applications of this technology should not be understated: in applications where neutron $/ \gamma$ discrimination is a priority, such as in portal monitoring for the presence of fissile material, extremely high levels of discrimination are required. Significant regions of overlap wherein events cannot be identified with sufficient
TABLE I

SiX-PARAMETER VALUES FOR MARRONE's MODEl FOR THE ACCURATE REPRODUCTION OF THE AVERAGE NEUTRON AND $\gamma$-RAY PULSE SHAPES ACQUIRED USING AN EJ-301 SCINTILLATOR

\begin{tabular}{ccccccc}
\hline Parameters & $A$ & $B$ & $\theta(\mathrm{ns})$ & $\lambda_{s}(\mathrm{~ns})$ & $\lambda_{l}(\mathrm{~ns})$ & $t_{0}(\mathrm{~ns})$ \\
\hline$\gamma$ ray & 10.53 & 0.017 & 4.368 & 3.482 & 11.52 & 0.331 \\
Neutron & 11.32 & 0.017 & 4.317 & 3.537 & 38.14 & 0.286 \\
\hline
\end{tabular}

confidence undermine the use of liquid organic scintillators in these applications. Furthermore, portability is an almost ubiquitous requirement of mixed-field instruments for use on plant and in inspection applications, and thus the deployment of discrimination methods on embedded platforms can be very desirable.

Detailed descriptions of FGA and its comparison with PGA are given in Sections 2 and 3; the verification process and experimental results are given in Section 4 and the discussion and the conclusions arising from this research are given in Sections 5 and 6.

\section{The PRINCIPLE OF THE FGA DisCRIMINATION SySTEM}

\section{A. Empirical Characterization of Organic Liquid Scintillators}

An empirical formulism for the characterization of a liquid scintillation detector for a given volume can provide a generic mathematical description of pulses from that detector which can be used to simulate the response from such a detector. The sixparameter function [5] that describes scintillator pulse shapes is henceforth referred to as Marrone's model in this work and is given by:

$$
\begin{aligned}
y(t)=A\left[\exp \left(-\frac{t-t_{0}}{\theta}\right)-\exp \right. & \left(-\frac{t-t_{0}}{\lambda_{s}}\right) \\
& \left.+B \exp \left(-\frac{t-t_{0}}{\lambda_{l}}\right)\right]
\end{aligned}
$$

where $A$ and $B$ are the amplitudes of the short (fast) and long (slow) components at $t=0$, respectively, $\lambda_{s}$ is the decay time constant for the short component, $\lambda_{l}$ is the decay time constant for the long component, $\theta$ is the third decay constant and $t_{0}$ is the time reference for the start of the signal. Table I provides the values for each of the six parameters for $\gamma$-ray and neutron pulse shapes, respectively, which are estimated from the data acquired using an EJ-301 scintillator [11]. A typical pulse shape according to (1) and Table I for each radiation type is given in Fig. 1.

As depicted in Fig. 1, the rising portion of a neutron pulse is almost identical to that of a $\gamma$-ray pulse, but the falling portions of their pulses are significantly different. Therefore, in order to decrease the complexity of the following analysis and to reduce the processing overhead, the rising portion is discarded and the pulse waveform is translated left by an amount $t_{m}$ so that the peak of the pulse is on the $y$-axis. Thus (1) can be expressed as,

$$
\begin{aligned}
x(t)= & y\left(t+t_{m}\right) \cdot u(t) \\
= & A\left[\exp \left(-\frac{t+t_{0}^{\prime}}{\theta}\right)-\exp \left(-\frac{t+t_{0}^{\prime}}{\lambda_{s}}\right)\right. \\
& \left.+B \exp \left(-\frac{t+t_{0}^{\prime}}{\lambda_{l}}\right)\right] \cdot u(t)
\end{aligned}
$$




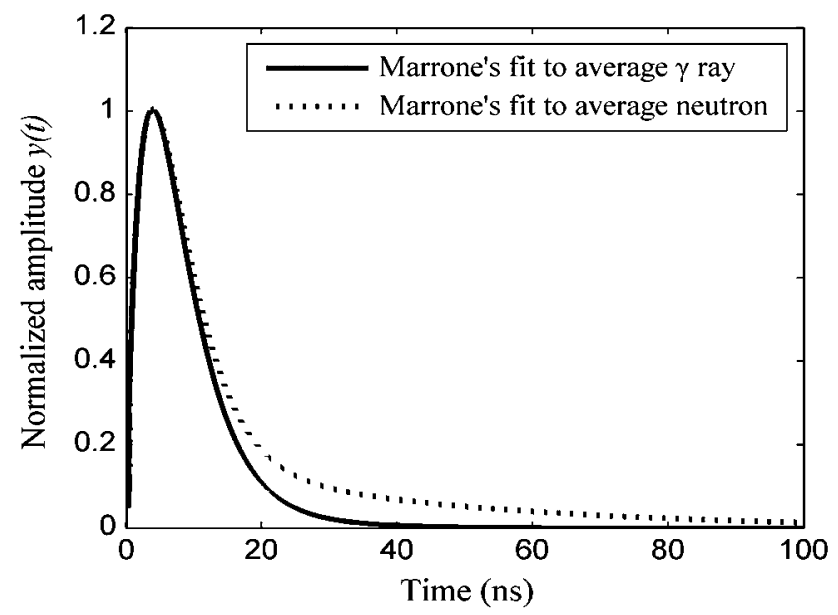

Fig. 1. A plot of normalized amplitude versus time for an average neutron (broken line) and $\gamma$-ray pulse shape (solid line).

where $t_{0}^{\prime}=t_{m}-t_{0}$. The values of $t_{m}$ and $t_{0}^{\prime}$ are $3.875 \mathrm{~ns}$ and $3.544 \mathrm{~ns}$ for neutron pulse, $4.000 \mathrm{~ns}$ and $3.714 \mathrm{~ns}$ for $\gamma$-ray pulse, respectively.

\section{B. The Theoretical Basis of FGA}

For signal processing purposes, the Fourier transform often takes a time series or a function of continuous time, and maps it onto a frequency spectrum [12]. That is, it takes a function from the time domain and transforms it into the frequency domain which is essentially a decomposition of a function into sinusoids of different frequencies. In the case of a Fourier series or discrete Fourier transform, the sinusoids are harmonics of the fundamental frequency of the function being analyzed. When the function $x(t)$ is a function of time and represents a physical signal, the transform has a standard interpretation as the frequency spectrum of the signal. The magnitude of the resulting complex-valued function $F(f)$ at frequency $f$ represents the amplitude of a frequency component whose initial phase is given by the phase of $F(f)$.

1) Feature Extraction: The Fourier transform of (2) is,

$$
\begin{aligned}
X(f) & =\mathrm{FT}(x(t))=\int_{-\infty}^{+\infty} x(t) e^{-\mathrm{i} 2 \pi \mathrm{ft}} d t \\
& =X_{1}(f)+\mathrm{i} \cdot X_{2}(f)
\end{aligned}
$$

where $X_{1}(f)$ and $X_{2}(f)$ are the real parts and imaginary parts of $X(f)$, respectively, and the magnitude spectrum of $x(t)$ is thus,

$$
|X(f)|=\sqrt{\left|X_{1}(f)\right|^{2}+\left|X_{2}(f)\right|^{2}} .
$$

Given the parameters in the Table I, the dependence of $|X(f)|$ with $f$ is shown in Fig. 2 for a $\gamma$ ray and neutron. There are distinct differences between the magnitude spectrum of the $\gamma$-ray event and the neutron event which can be used as prominent features to discriminate them. The frequency of the intersection of the two curves in this case is $13.8 \mathrm{MHz}$. Below this frequency, the amplitude of each frequency component of neutron pulse is greater than that of $\gamma$-ray pulse and the magnitude spectrum of neutron pulse decreases more sharply than that of $\gamma$-ray pulse; above this frequency, the magnitude spectra of both pulses have

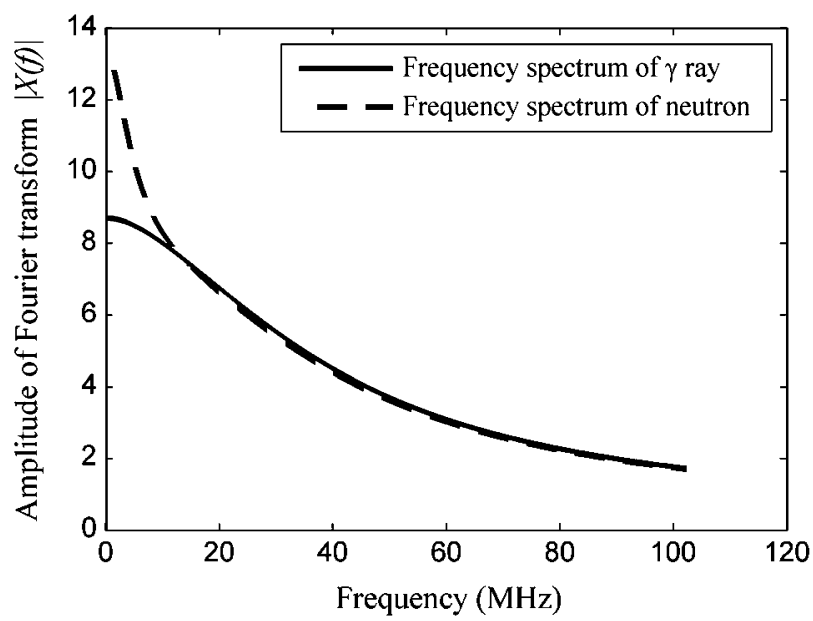

Fig. 2. The magnitude spectra of $\gamma$-ray events and neutron events. The differences between them are distinct and can be used to discriminate each other.

nearly the same amplitude. Therefore beyond the intersection they can no longer be discriminated on this basis.

The optimal discrimination parameter in this context is thus the value of the Fourier transform at zero frequency, $|X(0)|$ which is the average value of $x(t)$. Since the falling portion of neutron pulse decreases more slowly than that of $\gamma$-ray events, the average value for a neutron, $\left|X_{\mathrm{n}}(0)\right|$, is greater than that for $\gamma$-ray events, $\left|X_{\gamma}(0)\right|$, in the same time interval as expected intuitively.

In order to increase the FOM to take full advantage of the information provided by Fourier transform, the method of gradient analysis can be used to replace the single parameter $|X(0)|$. Like the PGA method, a frequency gradient analysis (FGA) is used here to discriminate neutron and $\gamma$-ray events. The discrimination parameter is for this purpose defined as,

$$
k_{f}(f)=\frac{|X(0)|-|X(f)|}{f}
$$

2) Feature Optimization: The FGA method uses this decay gradient between the amplitude of the zero-frequency component and the amplitude of the non-zero frequency component to discriminate neutron and $\gamma$-ray events. According to (2) and (5), the optimal $f$ can be obtained.

$$
\begin{aligned}
\Delta k_{f}(f)= & k_{f n}(f)-k_{f \gamma}(f) \\
= & \frac{\left|X_{\mathrm{n}}(0)\right|-\left|X_{\mathrm{n}}(f)\right|}{f} \\
& -\frac{\left|X_{\gamma}(0)\right|-\left|X_{\gamma}(f)\right|}{f}
\end{aligned}
$$

The relationship between $\Delta k_{f}(f)$ and frequency is shown in Fig. 3. At $f=4.6 \mathrm{MHz}, \Delta k_{f}(f)$ is a maximum. In reality, it is impossible to isolate this frequency because the above discussion assumes a continuous system in which the data length is infinitely long; in reality it is based on a discrete process and the associated data length is limited. The magnitude spectrum is obtained by Fast Fourier Transform (FFT) [12].

The sample frequency $F_{s}$ of our experimental system is 8 Giga Samples/Second (GSa/s) and the data length $N$ is usually 256 points giving a frequency resolution of $32 \mathrm{MHz}$. Combining 


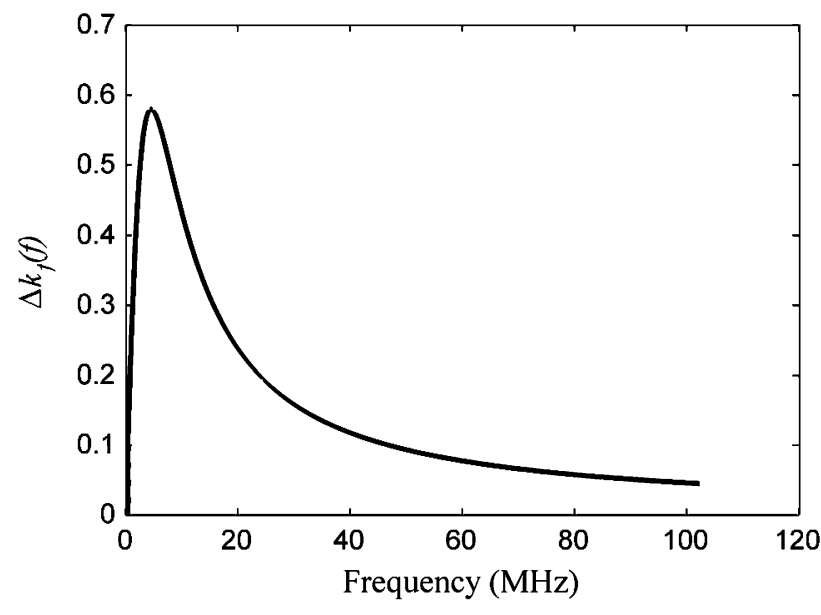

Fig. 3. The relationship between $\Delta k_{f}(f)$ and frequency.

this and insight from Fig. 3, we select the first frequency component $|X(1)|$ of the FFT as $|X(f)|$ in (5), which will obtain a suboptimal gradient. Now (5) becomes,

$$
k_{f}=\frac{N}{F_{s}}(|X(0)|-|X(1)|)
$$

On the basis of (7), the FGA method proposed here can be implemented. It is not necessary to calculate the amplitudes of all the frequency components of the FFT, because $X(0)$ and $X(1)$ can be calculated according to the following equations,

$$
\begin{aligned}
X(0)= & \sum_{n=0}^{N-1} x(n)=N \bar{x} \\
X(1)= & \sum_{n=0}^{N-1} x(n) \cos \left(\frac{2 \pi}{N} n\right) \\
& -\mathrm{i} \cdot \sum_{n=0}^{N-1} x(n) \sin \left(\frac{2 \pi}{N} n\right)
\end{aligned}
$$

where $\bar{x}$ is the mean value of $x(n)$. If the values of $\cos (2 \pi n / N)$ and $\sin (2 \pi n / N)$ are calculated in advance, $X(1)$ can be obtained quickly with a lookup table.

\section{THE COMPARISON OF FOM OF FGA AND PGA}

\section{A. An Introduction of FOM}

The FOM is a measure of the separation that can be achieved between different types of event distributions, and is calculated using [13],

$$
\mathrm{FOM}=\frac{S}{\mathrm{FWHM}_{\gamma}+\mathrm{FWHM}_{\mathrm{n}}}
$$

where $S$ is the separation between the peaks of the two events, $\mathrm{FWHM}_{\gamma}$ is the full-width-half-maximum of the spread of events classified as $\gamma$-ray events and $\mathrm{FWHM}_{\mathrm{n}}$ is the FWHM of the spread in the neutron peak. If the probability distribution of each event type is assumed to be a Gaussian distribution, (9) becomes,

$$
\mathrm{FOM}=\frac{\left|\mu_{\mathrm{n}}-\mu_{\gamma}\right|}{2.35\left(\sigma_{\gamma}+\sigma_{\mathrm{n}}\right)}
$$

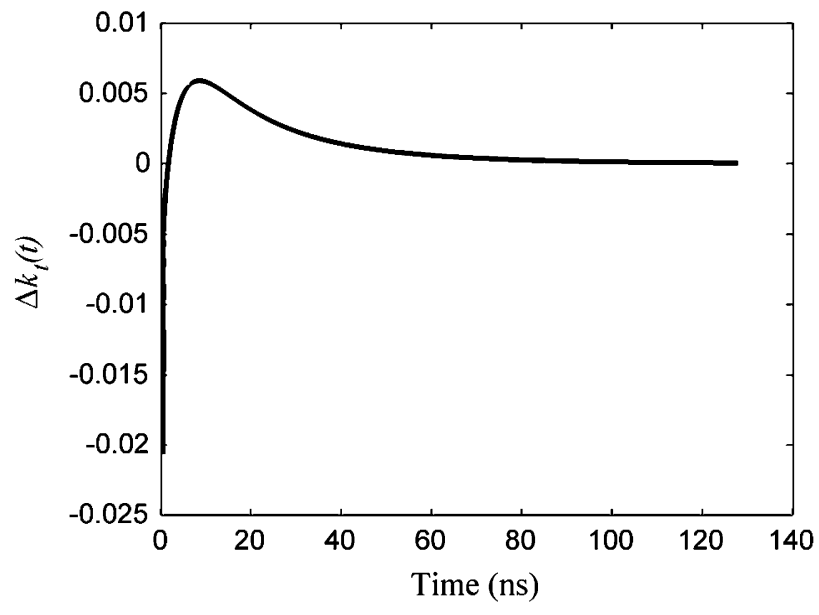

Fig. 4. The relationship between $\Delta k_{t}(t)$ and time. With the increase of time, $\Delta k_{t}(t)$ increases quickly and gets to a maximum, and then it decreases slowly.

where the mean, $\mu$, is given as $\mu_{\gamma}$ and $\mu_{\mathrm{n}}$ for the $\gamma$-ray and neutron Gaussians, respectively. The standard deviation, $\sigma$, is given as $\sigma_{\gamma}$ and $\sigma_{\mathrm{n}}$ for the $\gamma$-ray and neutron Gaussians, respectively.

\section{B. Pulse Gradient Analysis (PGA)}

The time-domain feature extracted by PGA is the gradient between peak amplitude and discrimination amplitude (the amplitude a specified time after the peak amplitude) [8]. The gradient is calculated using the following equation,

$$
k_{t}=\frac{\mathrm{d} x}{\mathrm{~d} t}=\frac{x_{\mathrm{d}}-x_{\mathrm{p}}}{t_{\mathrm{d}}-t_{\mathrm{p}}}
$$

where $k_{t}$ is the gradient in time domain, $x_{\mathrm{p}}$ is the peak amplitude, $x_{\mathrm{d}}$ is the discrimination amplitude, $t_{\mathrm{p}}$ is the time or sample that $x_{\mathrm{p}}$ occurred and $t_{\mathrm{d}}$ is the time or sample that $x_{\mathrm{d}}$ occurred. Further simplification of $k_{t}$ follows since only $x_{\mathrm{d}}$ varies once the pulse is normalized and thus (11) becomes,

$$
k_{t}=\frac{x_{\mathrm{d}}-1}{T}
$$

In PGA method this decay gradient is used to discriminate neutron and $\gamma$-ray events.

Combining (2) and (12), the optimal $T$ can be obtained,

$$
\begin{aligned}
\Delta k_{t}(t) & =k_{\mathrm{t} \gamma}(t)-k_{\mathrm{tn}}(t) \\
& =\frac{x_{\mathrm{d} \gamma}(t)-x_{\mathrm{dn}}(t)}{t}
\end{aligned}
$$

The relationship between $\Delta k_{t}(t)$ and $t$ is shown in Fig. 4. In this case, when $t=8.625 \mathrm{~ns}, \Delta k_{t}(t)$ is maximized. In experimental terms, this is largely-dependent on the scintillator decay time constants [14], [15]. Thus $T$ is usually selected within the range from $20 \mathrm{~ns}$ to $40 \mathrm{~ns}$. In the experimental system used in this research, according to the analysis results of FGA, $T=N / F_{s}=$ $256 / 8 \mathrm{GHz}=32 \mathrm{~ns}$ was selected.

\section{The FOM of the PGA Method}

In practical scenarios where the variation in pulse shape from the PMT makes a contribution, (2) becomes,

$$
s(t)=x(t)+n(t)
$$


where it is assumed that the variation is additive, and that $n(t)$ has a broad spectral response with a normal distribution, with a mean of zero and standard deviation of $\sigma$. Via (12), the mean value and standard deviation of $k_{t}(T)$ can be determined,

$$
\begin{aligned}
E\left[k_{t}(T)\right] & =\frac{1}{T}\left(1-x_{\mathrm{d}}(T)\right) \\
\operatorname{std}\left[k_{t}(T)\right] & =\frac{1}{T} \sigma .
\end{aligned}
$$

In the above derivation, it is assumed that $x_{\mathrm{d}}(T)$ is a constant and the pulse variation in shape $n(t)$ is independent on $x(t)$. According to (15), the FOM of PGA method is hence,

$$
\begin{aligned}
\mathrm{FOM}_{\mathrm{PGA}} & =\frac{E\left[k_{t_{\gamma}}(T)\right]-E\left[k_{t_{\mathrm{n}}}(T)\right]}{2.35\left(\operatorname{std}\left(k_{t_{\gamma}}(T)\right)+\operatorname{std}\left(k_{t_{\mathrm{n}}}(T)\right)\right)} \\
& =\frac{x_{\mathrm{dn}}(T)-x_{\mathrm{d} \gamma}(T)}{2.35\left(\sigma_{\gamma}+\sigma_{\mathrm{n}}\right)} .
\end{aligned}
$$

\section{The FOM of the FGA Method}

Similarly, we can calculate the performance parameters of FGA according to (7). In order to simplify the derivation, $|X(N / 2)|$ is used to replace $|X(1)|$ and it is assumed $|X(N / 2)|=0$, which is plausible according to Fig. 3. So (7) can be rewritten as,

$$
k_{f}=\frac{|X(0)|}{F_{s} / 2}
$$

According to (8), expressions for the mean value and standard deviation of $k_{f}$ can be determined as,

$$
\begin{aligned}
E\left[k_{f}\right] & =\frac{2}{F_{s}} N \bar{x} \\
\operatorname{std}\left[k_{f}\right] & =\frac{2}{F_{s}} \sqrt{N} \sigma .
\end{aligned}
$$

In this derivation, it is assumed that $\bar{x}$ is a constant and the pulse variance $n(t)$ is independent of $x(t)$. Thus according to (18), the FOM of the FGA method is,

$$
\begin{aligned}
\operatorname{FOM}_{\mathrm{FGA}} & =\frac{E\left[k_{\mathrm{fn}}\left(F_{s} / 2\right)\right]-E\left[k_{\mathrm{f} \gamma}\left(F_{s} / 2\right)\right]}{2.35\left(\operatorname{std}\left(k_{f_{\mathrm{n}}}\left(F_{s} / 2\right)\right)+\operatorname{std}\left(k_{f_{\gamma}}\left(F_{s} / 2\right)\right)\right)} \\
& =\frac{\sqrt{N}}{2.35} \cdot \frac{\left(\bar{x}_{\mathrm{n}}-\bar{x}_{\gamma}\right)}{\left(\sigma_{\gamma}+\sigma_{\mathrm{n}}\right)}
\end{aligned}
$$

\section{E. Comparison of FOM}

To compare the performance between PGA and FGA, the ratio of (19) to (16) is taken, to give,

$$
k_{\mathrm{FOM}}(N)=\sqrt{N} \frac{\bar{x}_{\mathrm{n}}-\bar{x}_{\gamma}}{x_{\mathrm{dn}}\left(N / F_{s}\right)-x_{\mathrm{d} \gamma}\left(N / F_{s}\right)} .
$$

Since the PGA method usually uses a moving average filter to obtain $x_{\mathrm{dn}}(T)$ and $x_{\mathrm{d} \gamma}(T)$ and, assuming the number of points in the average filter is $M, M \leqslant N$, and following a similar derivation to that of (19), (16) becomes,

$$
\mathrm{FOM}_{\mathrm{PGA}}^{\prime}=\sqrt{M} \frac{x_{\mathrm{dn}}(T)-x_{\mathrm{d} \gamma}(T)}{2.35\left(\sigma_{\gamma}+\sigma_{\mathrm{n}}\right)}=\sqrt{M} \mathrm{FOM}_{\mathrm{PGA}}
$$

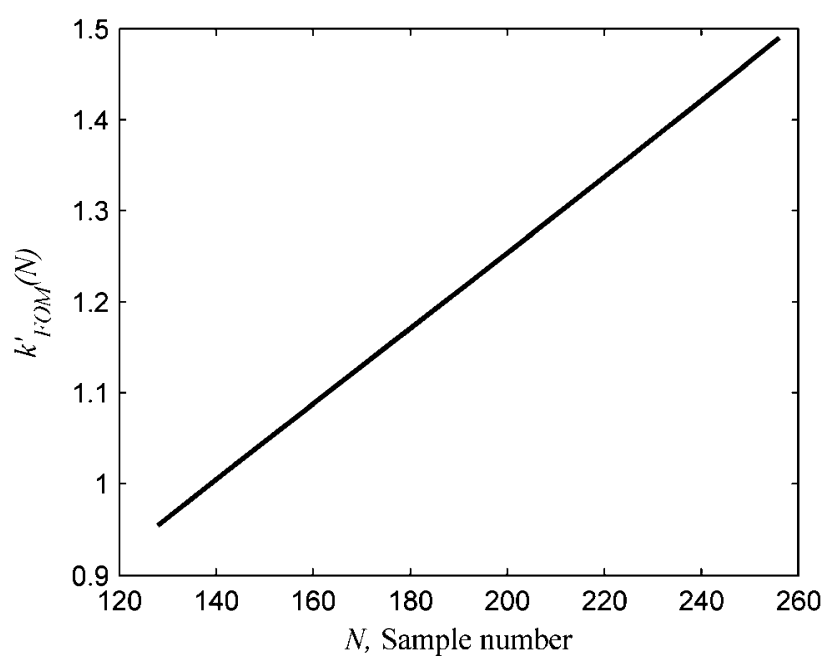

Fig. 5. The variation of the discrimination performance ratio $k_{\mathrm{FOM}}^{\prime}(N)$ with sample number $N$. With the increase of sample number, $k_{\mathrm{FOM}}^{\prime}(N)$ becomes larger.

and thus (20) can be revised as,

$$
k_{\mathrm{FOM}}^{\prime}(N)=\sqrt{\frac{N}{M}} \frac{\bar{x}_{\mathrm{n}}-\bar{x}_{\gamma}}{x_{\mathrm{dn}}\left(N / F_{s}\right)-x_{\mathrm{d} \gamma}\left(N / F_{s}\right)} .
$$

For the case when $M=N / 3$, and using (2), the dependence of $k_{\mathrm{FOM}}^{\prime}(N)$ with $N$ is shown in Fig. 5. With the increase of number of samples or of the discrimination time, it is clear that the discrimination performance of FGA is better than that of PGA. In particular, at $N=256, k_{\mathrm{FOM}}^{\prime}=1.46$.

\section{EXPERIMENTAL}

\section{A. Experimental Setup}

The experimental data analyzed in this work were acquired using the TOF measurement system at the National Physical Laboratory, Teddington, U.K. (this set-up and its use in acquiring the data considered in this work is described in detail in [16]). The ${ }^{7} \mathrm{Li}(p, n){ }^{7} \mathrm{Be}$ reaction was used to provide TOF data for neutrons at two energies $0.745 \mathrm{MeV}$ and $1.225 \mathrm{MeV}$. The detector system for the digital acquisition consisted of a $4.5 \mathrm{ml}$ cylindrical cell scintillation detector filled with EJ-301 organic liquid, optically-coupled to a Hamamatsu R5611 photomultiplier tube (PMT). The PMT was operated with a negative supply voltage of $-840 \mathrm{~V}$ DC. The output signal from the scintillator was connected to channel 1 of an Infinium ${ }^{\circledR}$ digital oscilloscope, via approximately $30 \mathrm{~m}$ of high bandwidth cable. The beam-pickup signal was connected to a leading-edge discriminator in the control room, and the discriminator output was then passed (via a delay) to another input of the digital oscilloscope. Scintillator pulses were used to trigger acquisition.

The scintillator pulse and the corresponding beam-pickup pulse data were captured digitally with a sampling rate of $8 \mathrm{GSa} / \mathrm{s}$ and 16-bit amplitude resolution. This enabled all detected events, i.e., both $\gamma$ rays and neutrons, to be sorted in terms of their time-of-arrival relative to the initial beam-pickup input of the digital oscilloscope. 

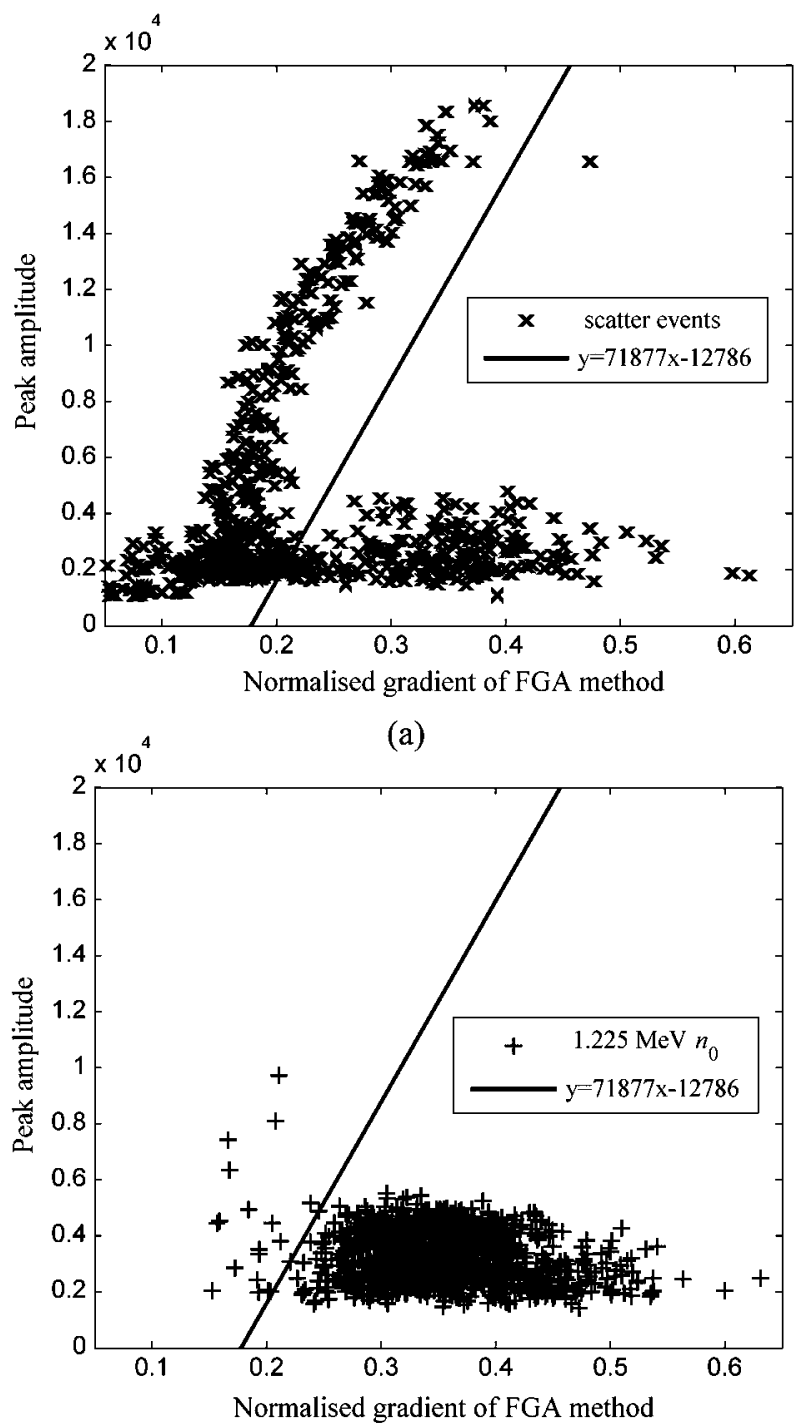

(c)

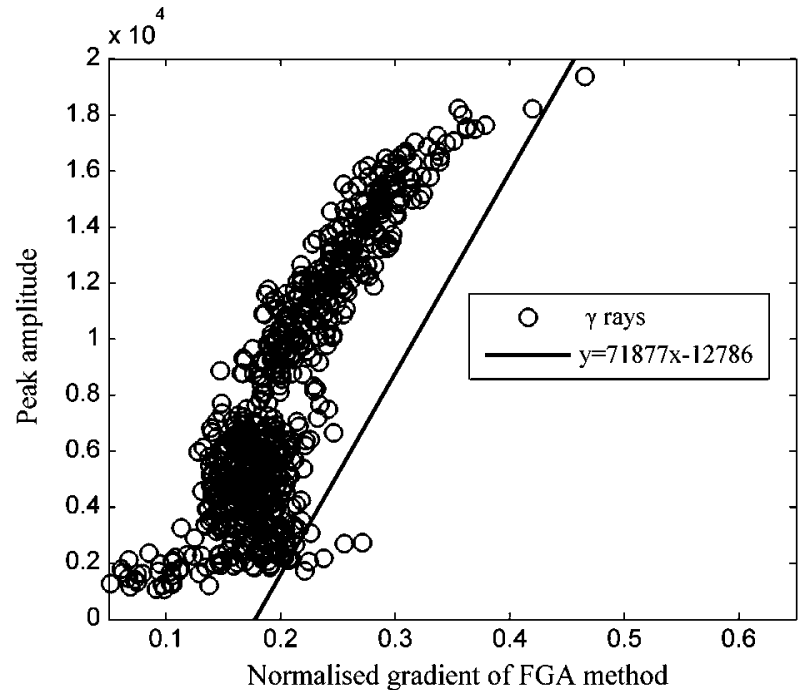

(b)

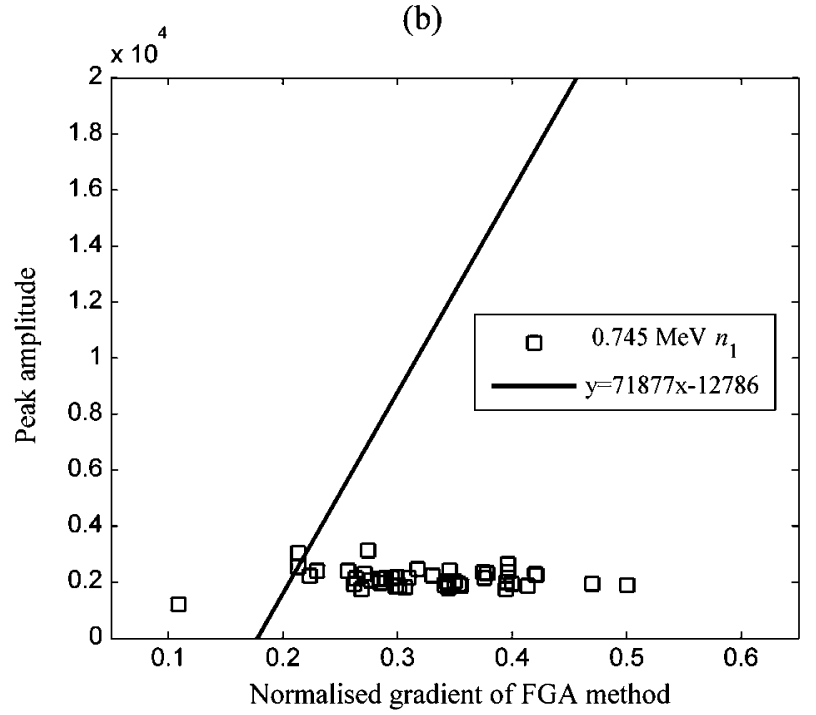

(d)

Fig. 6. Scatter plots of peak amplitude against the $32 \mathrm{~ns}$ ( 256 data) normalized gradient of the FGA method for the four event types that comprise the total mixed field distribution studied in this work. The TOF spectrum has been used to identify $\gamma$ rays, $1.225 \mathrm{MeV}$ neutrons $\left(n_{0}\right), 0.745 \mathrm{MeV}$ neutrons $\left(n_{1}\right)$ and scattered events, as denoted by the symbol of the events in each case. The straight line in each subplot is the discrimination line established on the basis of the centroids of the clusters. An event is a $\gamma$-ray event or neutron event is determined by whether the event is above or below the straight line, respectively. (a) Scatter events discriminated by TOF and FGA; (b) $\gamma$-ray events discriminated by TOF and FGA; (c) $1.225 \mathrm{MeV}$ neutrons discriminated by TOF and FGA; (d) $0.745 \mathrm{MeV}$ neutrons discriminated by TOF and FGA.

\section{B. Experimental Results}

1) Verification of FGA by TOF: TOF spectral analysis is a reliable means of discriminating $\gamma$-ray and neutron events, and for neutron spectrometry, so it is very helpful to use TOF method to develop and investigate different discrimination methods. Three specific kinds of radiation types can be identified according to TOF method: $\gamma$ rays, and neutrons of energies $n_{0}(1.225 \mathrm{MeV})$ and $n_{1}(0.745 \mathrm{MeV})$. In addition, there are those classified as scattered events. These arise as a result of scatter in the environment and within the detector and fall outside of the TOF assignments of the three main event types.

The $\gamma$ rays and neutron events and thus masked in terms of their TOF and this provides an alternative, independent basis for the identification of radiation type alongside PSD. Each event has been denoted by a symbol corresponding to its TOF assignment and this has been folded into the scatter plot achieved on the basis of the FGA method. The results of this analysis are shown in Fig. 6 for each event type. The threshold for discrimination is depicted by a semi-arbitrary line on each plot to guide the eye. This is determined on the basis of an established technique [17] whereby the centroid of each cluster of data is identified and a linear fit is applied to the midpoints between the clusters.

In Fig. 6(a) for example, the scatter events are shown, with a distribution that extends throughout the event plane indicating the lack of discrimination possible with TOF for these events. In Fig. 6(b), the result for the $\gamma$ rays is given, with a significant concentration of events in the low-gradient area of the event plane. In Figs. 6(c) and (d) the corresponding results for the 


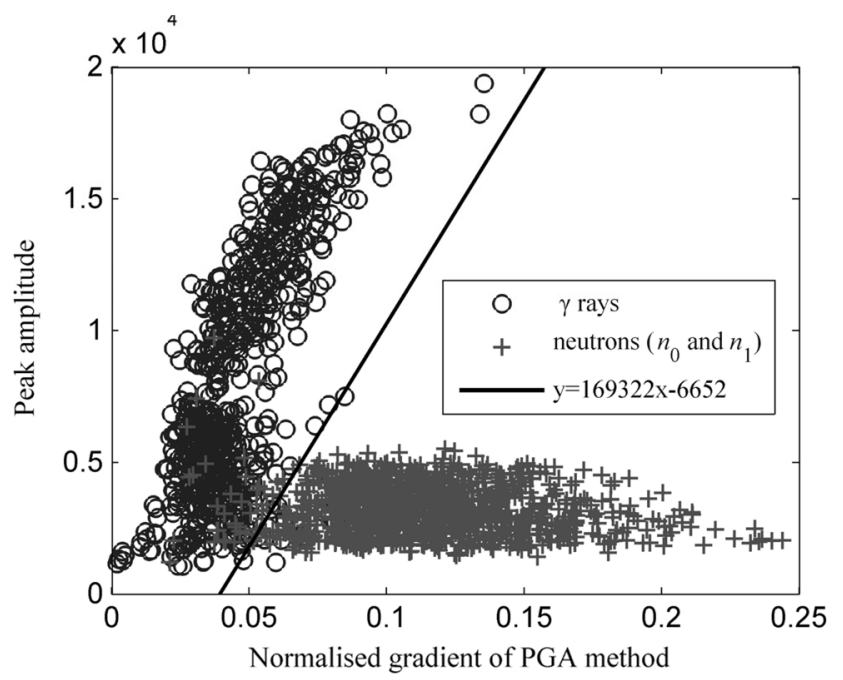

(a)

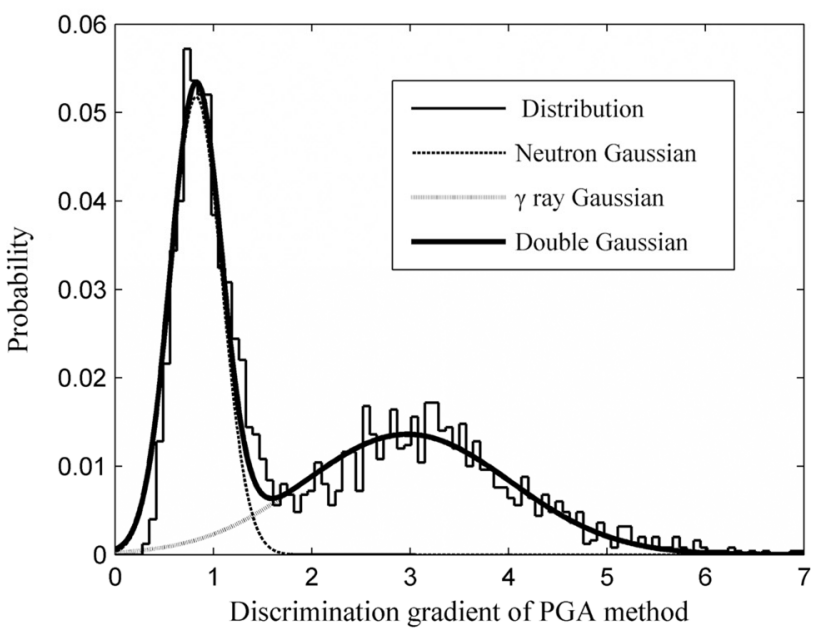

(b)

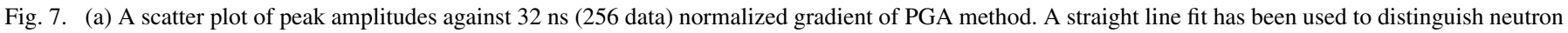

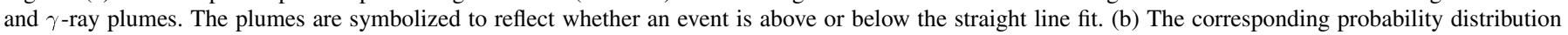
histogram for the PGA data with fitted Gaussian distributions.

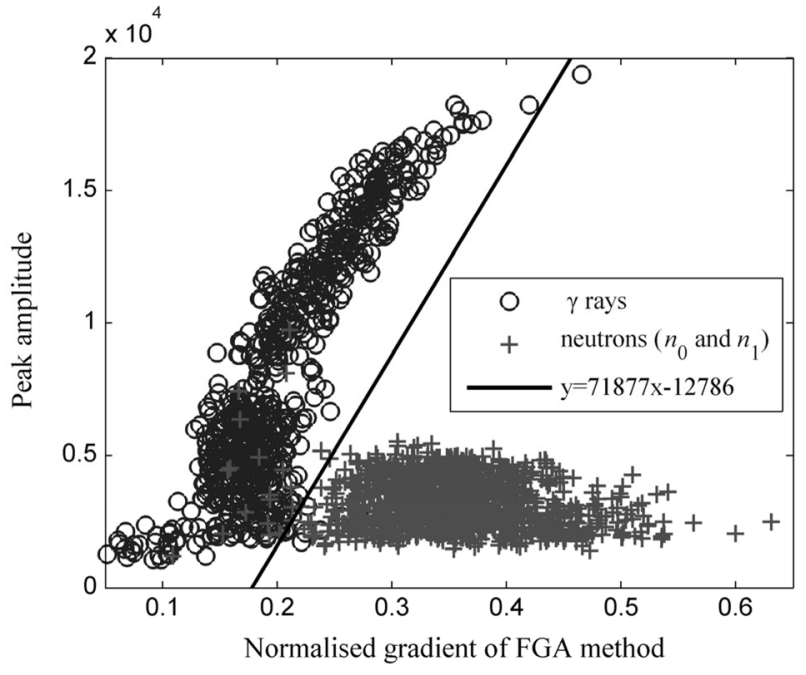

(a)

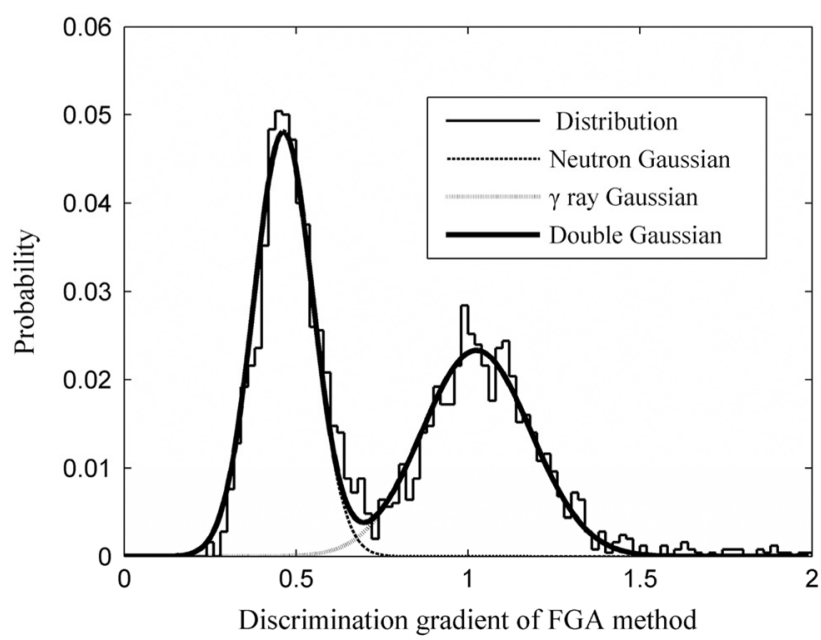

(b)




histogram for the FGA data with fitted Gaussian distributions.

neutrons are given, indicating the corresponding response with most events in the high-gradient region of the event plain. These results provide a qualitative basis confirming that FGA provides a good degree of discrimination for the mixed field explored in this research.

2) FOMs of FGA and PGA: The scatter plots of peak amplitude against the $32 \mathrm{~ns}$ (256 data) normalized gradient of both the PGA and FGA methods, and their corresponding probability distribution histograms, are given in Figs. 7 and 8, respectively. In Figs. 7(a) or 8(a), two plumes are evident separated in terms of normalized gradient of PGA or FGA, relative to peak amplitude, which correspond to $\gamma$-ray and neutron events. It is also clear that the level of discrimination with FGA is better than that of PGA, which is verified by the calculation of the FOM in each case as shown in Table II. Gaussian fits have been ap- plied to the experimental distributions shown in Figs. 7(b) and 8 (b) using the curve fitting tool available in MATLAB ${ }^{\circledR}$. The Gaussian distribution is expressed as,

$$
f(x)=A \exp \left[-\frac{(x-\mu)^{2}}{2 \sigma^{2}}\right]
$$

and the sum of Gaussian distributions as,

$$
f(x)=A_{\gamma} \exp \left[-\frac{\left(x-\mu_{\gamma}\right)^{2}}{2 \sigma_{\gamma}^{2}}\right]+A_{\mathrm{n}} \exp \left[-\frac{\left(x-\mu_{\mathrm{n}}\right)^{2}}{2 \sigma_{\mathrm{n}}^{2}}\right]
$$

The Gaussian functions are scaled using $A_{\gamma}$ and $A_{\mathrm{n}}$ for the $\gamma$-ray and neutron Gaussians, respectively, and other symbols in 
TABLE II

The VAlues of PARAMETERS IN (27) CALCUlated From the EXPERIMENTAL DATA BY USING PGA AND FGA, RESPECTIVELY, AND THEIR CORRESPONDING FOMS CALCULATED WITH (14)

\begin{tabular}{cccccccc}
\hline Method & $A_{\gamma}$ & $\mu_{\gamma}$ & $\sigma_{\gamma}$ & $A_{\mathrm{n}}$ & $\mu_{\mathrm{n}}$ & $\sigma_{\mathrm{n}}$ & $F O M$ \\
\hline PGA & 0.0136 & 2.97 & 1.047 & 0.0517 & 0.823 & 0.259 & 0.699 \\
FGA & 0.0233 & 1.02 & 0.158 & 0.0481 & 0.462 & 0.0851 & 0.981 \\
\hline
\end{tabular}

TABLE III

EXPERIMENTAL RESULTS OF THE RATIOS OF COUNTS OF EACH TYPE TO THE TOTAL FOR TOF, PGA AND FGA. THERE ARE A TOTAL OF 2500 EVENTS INCLUDING SCATTER, AND 1821, 1766 AND 1795 WiTHOUT SCATTER, RESPECTIVELY

\begin{tabular}{|c|c|c|c|c|c|c|c|}
\hline Method & $n_{0}$ & $n_{1}$ & $n_{\text {total }}$ & $\gamma$ rays & Scatter & $n_{\text {total }} / \gamma$ & $F O M$ \\
\hline $\begin{array}{l}\text { TOF } \\
\text { Error }\end{array}$ & $\begin{array}{c}0.43 \\
+0.01\end{array}$ & $\begin{array}{c}0.017 \\
+0.003\end{array}$ & $\begin{array}{c}0.45 \\
+0.01\end{array}$ & $\begin{array}{c}0.28 \\
+0.0\end{array}$ & $\begin{array}{c}0.27 \\
+0.01\end{array}$ & $\begin{array}{l}1.61 \\
+0.07\end{array}$ & \multirow[t]{2}{*}{ - } \\
\hline & & -0.00 & -0.01 & -0.01 & -0.01 & -0.07 & \\
\hline $\begin{array}{l}\text { PGA } \\
\text { Error }\end{array}$ & - & - & 0.59 & 0.41 & - & 1.44 & \multirow[t]{2}{*}{0.699} \\
\hline Error & - & - & \pm 0.02 & \pm 0.01 & - & \pm 0.06 & \\
\hline FGA & - & - & 0.54 & 0.46 & - & 1.17 & \multirow{2}{*}{0.981} \\
\hline Error & - & - & \pm 0.015 & \pm 0.014 & - & \pm 0.05 & \\
\hline
\end{tabular}

(24) are the same as those in (10). Table II presents the means, standard deviations and $A_{\gamma}$ and $A_{\mathrm{n}}$ for the Gaussian fits to the experimental data shown in Figs. 7(b) and 8(b). According to the results of Gaussian fits and (10), the FOMs of PGA and FGA and their ratio can be calculated as,

$$
\begin{aligned}
\mathrm{FOM}_{\mathrm{PGA}}^{\prime}=0.699, \mathrm{FOM}_{\mathrm{FGA}}=0.981 \\
k_{\mathrm{FOM}}^{\prime}=1.40
\end{aligned}
$$

3) Collection of Experimental Results: The experimental results of TOF, PGA and FGA are shown in Table III, some of which have been given in literature [16] and are repeated here for clarity. The TOF data were obtained by fitting the three components of the TOF spectrum [16] with a Gaussian expression and then limits either side of each component were applied at the $10 \%$ level of the peak amplitude. The temporal information that is obtained from the TOF data enables each component in a field under study to be isolated, i.e., each neutron energy component, $\gamma$-ray component and the scattered components. The PGA and FGA methods provide the PSD. Therefore, only the total neutron component and $\gamma$-ray component are included in Table III for PGA and FGA. The ratios of neutron to $\gamma$-ray component have also been calculated and are included in Table III, along with uncertainties corresponding to one standard deviation from the mean.

TOF analysis can identify those events that have scattered prior to detection, since most of these fall outside of the peaks for event classification, but it cannot classify scattered events specifically as neutron events or $\gamma$ rays. Conversely, PSD methods such as PGA and FGA cannot identify whether individual events have scattered but just that they are either $\gamma$-rays or neutron events from the differences in pulse shape. Therefore, in order to verify and compare the performances of PGA and FGA, the scattered events have been removed leaving only the $\gamma$ rays, $1.225 \mathrm{MeV}$ neutrons $\left(n_{0}\right)$ and 0.745
TABLE IV

THE Number of EACH Event IDENTIFIED By PGA AND FGA With THE HELP OF TOF

\begin{tabular}{cccccc}
\hline Methods & $n_{0}$ & $n_{1}$ & $n_{\text {total }}$ & $\gamma$ rays & $n_{\text {total }} / \gamma$ \\
\hline TOF & 1080 & 44 & 1124 & 697 & 1.61 \\
PGA & 1046 & 40 & 1086 & 680 & 1.60 \\
FGA & 1062 & 42 & 1104 & 691 & 1.60 \\
\hline
\end{tabular}

$\mathrm{MeV}$ neutrons $\left(n_{1}\right)$. Table IV shows the number of each event identified by PGA and FGA with the help of TOF.

\section{DISCUSSION}

\section{A. Experimental Data Arising From TOF Measurements}

It is clear from the data in Fig. 6 that the identification of events made on the basis of FGA is consistent with that performed independently by digital TOF. The $\gamma$-ray events are located as expected in the upper lobe of the scatter plot and the neutron events are located in the lower lobe. Furthermore, the discrimination of the neutron portion according to energy also agrees qualitatively with intuition; the $1.225 \mathrm{MeV}$ neutrons occupy the entire locus of the neutron lobe whilst the $0.745 \mathrm{MeV}$ neutrons are limited by comparison, both by peak and sample amplitude. The results obtained from Fig. 6 are also consistent with that of previous reports [16].

The background event data are spread evenly across both plumes in the scatter plot, some of which correspond to events that lie outside of the TOF windows, often as a result of scatter. Although the neutron $/ \gamma$-ray ratio of TOF is greater than that of FGA, the imbalance is consistent with the additional contribution of scatter events that cannot be discerned with FGA.

There are some events that are erroneously classified by the FGA method, and these are evident in Fig. 6. For example, some $\gamma$-ray events are incorrectly tagged by TOF measurement because a neutron interacting in the detector can, in principle, stimulate the production of a $\gamma$ ray which is subsequently detected; hence a neutron event is tagged as such by TOF but is manifest with a $\gamma$-ray pulse shape. Such events therefore occupy the low-gradient $\gamma$-ray region of the event plane in Fig. 6(c) and (d). Conversely, some $\gamma$-ray events are mistakenly identified as neutrons by the TOF method is because they arrive at the detector late, relative to directly-detected events, as a result of scatter. A few of these fall within the window applied to isolate the neutrons, and thus they are identified as such in Fig. 6(b).

\section{B. Performance Comparison of FGA and PGA}

Both the FGA method and the PGA method use gradient analysis to discriminate $\gamma$-ray events and neutron events, but each gradient belongs to different domain. The gradient used by PGA is defined as the difference between the peak amplitude and the discrimination amplitude, which is a feature being extracted from the time domain; while the gradient used by FGA is defined as the difference between the zero-frequency component and the first frequency component of Fourier transform of the acquired signal, which is a feature extracted from the frequency domain. In Section 3, it has been proven on a theoretical basis that the performance of FGA is better than that of PGA since 
PGA is very sensitive to noise and variations of the light intensity from the detector. FGA is less sensitive to the high-frequency components responsible for the variation in pulse shape.

The comparison between the FOMs of the FGA method and PGA method is reported in Table II and according to (25), the ratio of FOM between FGA and PGA is 1.40, which is consistent with the calculations achieved by (22) and Fig. 5 .

According to the data in Table IV, although the ratios of neutrons to $\gamma$ rays for PGA and FGA are nearly the same as that of TOF, there are some slight differences in the numbers of each event identified by them and the experimental results obtained from FGA are slightly closer to those of TOF. For example, the total number of $\gamma$-ray events arising from TOF, PGA and FGA are 697, 680 and 691, respectively. There are $17 \gamma$-ray events mistakenly classified as neutron events by PGA, $(2.4 \pm 0.6) \%$, whilst there are only $7 \gamma$-ray events being mistakenly classified as neutron events by FGA, $(1.0 \pm 0.3) \%$.

The results obtained in Figs. 7 and 8 with the PGA method and FGA algorithm applied to the data from the measurement of TOF show similar levels of performance. However, the overlap between the $\gamma$-ray events and neutron events in Fig. 8 have been dramatically decreased compared to that in Fig. 7. A further interesting phenomenon is that the neutron events in Fig. 8(a) are more closely congregated than that in Fig. 7(a), which is reflected by the significantly reduced $\sigma_{n}$ for FGA (0.085) compared with PGA (0.259). This provides another perspective on the benefits of the FGA over the PGA method.

\section{CONCLUSION}

A wide variety of pulse-shape discrimination (PSD) methods have been successfully utilized to classify neutron and $\gamma$-ray events according to the excellent PSD properties and fast timing performance of liquid organic scintillation detectors. Most of these techniques exploit the time-domain features of the signal, which can render these methods sensitive to variation in the pulse shape response of the detection system.

Compared to time-domain discrimination methods, methods developed in the frequency domain are more robust to variations in pulse-shape response of the scintillant cell and the PMT. In this paper, a new PSD method based on the Fourier transform, called frequency gradient analysis (FGA) is proposed. This uses the difference between the zero-frequency component and the first frequency component of Fourier transform of the acquired signal. The objective of this method is to combine the advantage of insensitivity to pulse-shape variation of spectral analysis with that of real-time implementation of PGA algorithm.

Some measures have been taken to probe the feasibility of FGA for the digital discrimination of neutrons and $\gamma$ rays in mixed radiation fields. Firstly, an accepted empirical model has been used to deduce the discrimination ability and performance of FGA, which provides a theoretic basis of this method. Secondly, FGA method is verified against that achieved via the digital measurement of TOF. Thirdly, although discrimination afforded by the FGA method is observed to be consistent with that achieved by digital TOF, further research is necessary with regard to potential industrial applications. Such investigations might include, for example, to determine whether the FGA method is compatible with the capabilities of current embedded systems, whether it offers a degree of immunity to pile-up and whether it heralds a real-time means of PSD. The PGA method has been demonstrated to have these advantages and has been verified by TOF, and thus has been used in commercial instruments. In this work it has been shown that FGA method not only has some of the merits of PGA but also demonstrates a prominent improvement in FOM.

Perhaps the most pertinent attribute with regard to deployment on embedded systems is the issue of computational speed. PGA was designed with this as an explicit requirement, and yet does not discriminate successfully without normalization which requires a moving average filter. This clearly reduces processing speed. Conversely, FGA does not require explicit normalization but it is more computationally laborious than PGA. Clearly, as configurable embedded systems continue to develop in capability and speed, the relative merits of these methods will be dependent on the elegance with which they are implemented.

Since FGA provides a fast and stable digital technique for discriminating neutron and $\gamma$-ray events arising from organic scintillation materials, such as EJ-301, BC501A, and NE213, it is likely that these would be used most often in tandem with the FGA method. New materials are being developed to offer improved response and better materials characteristics, such as reduced flammability. The FGA method holds great potential for digital processing of data from detectors using these materials.

\section{ACKNOWLEDGMENT}

The authors would like to gratefully acknowledge the help and advice of Dr. David Thomas and Dr. Nigel Hawkes and the technical team at the National Physical Laboratory, Teddington, U.K.

\section{REFERENCES}

[1] G. F. Knoll, Radiation Detection and Measurement. New York: Wiley, 2000, pp. 230-231.

[2] F. D. Brooks, "A scintillation counter with neutron and gamma-ray discriminators," Nucl. Instrum. Methods, vol. 4, no. 3, pp. 151-163, Apr. 1959.

[3] T. K. Alexander and F. S. Goulding, "An amplitude-insensitive system that distinguishes pulses of different shapes," Nucl. Instrum. Methods, vol. 13, pp. 244-246, Aug. 1961.

[4] N. V. Kornilov, V. A. Khriatchkov, M. Dunaev, A. B. Kagalenko, N. N. Semenova, V. G. Demenkov, and A. J. M. Plompen, "Neutron spectroscopy with fast waveform digitizer," Nucl. Instrum. Methods Phys. Res. A, vol. A497, no. 2-3, pp. 467-478, Feb. 2003.

[5] S. Marrone et al., "Pulse shape analysis of liquid scintillators for neutron studies," Nucl. Instrum. Methods Phys. Res. A, vol. A490, no. 1-2, pp. 299-307, Sep. 2002.

[6] G. Liu, M. D. Aspinall, X. Ma, and M. J. Joyce, "An investigation of the digital discrimination of neutrons and $\gamma$ rays with organic scintillation detectors using an artificial neural network," Nucl. Instrum. Methods Phys. Res. A, vol. A607, no. 3, pp. 620-628, Aug. 2009.

[7] I. D'Antone, Fuzzy Techniques for Pulse Discrimination and Track Construction Apr. 1996 [Online]. Available: http://www-ceb.bo.infn.it/ docum/nt9604id/trk-fuceb_abs.html, CEB/NT-9604

[8] B. D'Mellow, M. D. Aspinall, R. O. Mackin, M. J. Joyce, and A. J. Peyton, "Digital discrimination of neutrons and $\gamma$ rays in liquid scintillators using pulse gradient analysis," Nucl. Instrum. Methods Phys. Res. A, vol. A578, no. 1, pp. 191-197, Jul. 2007.

[9] M. J. Joyce, M. D. Aspinall, F. Cave, K. Georgopoulos, and Z. Jarrah, "The design, build and test of a digital analyzer for mixed radiation fields," in Proc. Advances in Nuclear Instrumentation Measurement and Methods, Marseille, France, Jun. 2009. 
[10] S. Yousefi, L. Lucchese, and M. D. Aspinall, "Digital discrimination of neutrons and gamma-rays in liquid scintillators using wavelets," Nucl. Instrum. Methods Phys. Res. A, vol. A598, no. 2, pp. 551-555, Jan. 2007.

[11] M. D. Aspinall, B. D’Mellow, R. O. Mackin, M. J. Joyce, Z. Jarrah, and A. J. Peyton, "The empirical characterization of organic liquid scintillation detectors by the normalized average of digitized pulse shapes," Nucl. Instrum. Methods Phys. Res. A, vol. A578, no. 1, pp. 261-266, Jul. 2007.

[12] J. G. Proakis and D. G. Manolakis, Digital Signal Processing: Principles, Algorithms, and Applications. Upper Saddle River, NJ: Prentice-Hall, 1996, ch. 4.

[13] R. A. Winyard, J. E. Lukin, and B. W. McBeth, "Pulse shape discrimination in inorganic and organic scintillators," Nucl. Instrum. Methods, vol. 95 , no. 1 , pp. 141-153, Aug. 1971.
[14] Z. W. Bell, "Tests on a digital neutron-gamma pulse shape discriminator with NE213," Nucl. Instrum. Methods Phys. Res., vol. 188, no. 1 , pp. 105-109, 1981.

[15] Z. W. Bell, K. P. Ziock, M. F. Ohmes, Y. Xu, T. J. Downar, and S. A. Pozzi, "Measurement of neutron yields from $\mathrm{UF}_{4}$," in Proc. Nuclear Science Symp., Dresden, Germany, 2008, Paper N47-5.

[16] M. D. Aspinall, B. D’Mellow, R. O. Mackin, M. J. Joyce, N. P. Hawkes, D. J. Thomas, Z. Jarrah, A. J. Peyton, P. J. Nolan, and A. J. Boston, "Verification of the digital discrimination of neutrons and $\gamma$ rays using pulse gradient analysis by digital measurement of time of flight," Nucl. Instrum. Methods Phys. Res. A, vol. A583, no. 2-3, pp. 432-438, Dec. 2007.

[17] M. D. Aspinall, "Real Time Digital Assay of Mixed Radiation Fields," Ph.D. dissertation, Lancaster Univ., Lancaster, U.K., 2007. 